\title{
Thermally Functional Liquid Crystal Networks by Magnetic Field Driven Molecular Orientation
}

\author{
Jungwoo Shin, Minjee Kang, Tsunghan Tsai, Cecilia Leal, Paul V. Braun and David G. \\ Cahill*, \\ ${ }^{\dagger}$ Department of Materials Science and Engineering, University of Illinois at Urbana-Champaign, \\ Urbana, \\ Illinois 61801, USA.
}

Aligned liquid crystal networks (LCNs) were synthesized by mixing liquid crystal (LC) monomers (1,4-bis-[4-(3-acryloyloxypropyloxy)benzoyloxy]-2-methylbenzene; RM257, Wilshire Technologies) with $5 \mathrm{~mol} \%$ of cross-linker (pentaerythritol tetrakis[3mercaptopropionate]; PETMP, Sigma-Aldrich) and photoinitiator (2-hydroxy-2methylpropiophenone; $0.3 \mathrm{wt} \%$, Sigma-Aldrich). Substrates for TDTR measurements were prepared by spin-coating with polyimide (PI) film followed by Al thin film deposition by magnetron sputtering on sapphire substrates. Figure S1 shows the coating procedure: i) the LC solution was first dropped onto the glass slide; ii-iii) the glass slide was placed onto the $\mathrm{Al} / \mathrm{PI} / \mathrm{sapphire}$ substrate; iv) and the glass slide was used to cast a LC film across the $\mathrm{Al} / \mathrm{PI} / \mathrm{sapphire}$ substrate.

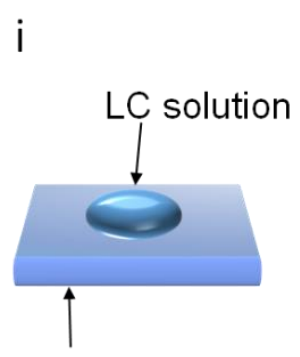

Glass slide

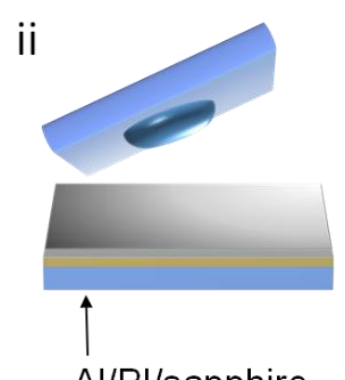

Al/Pl/sapphire iii

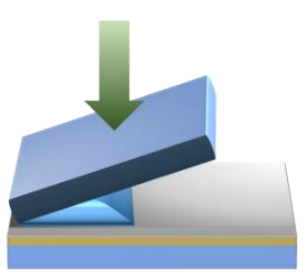

iv

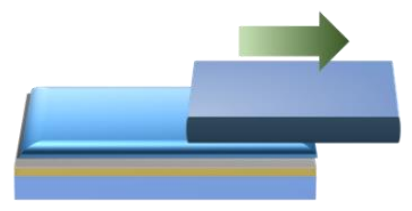

Figure S1. Schematic illustration for casting a LC solution on an A1/PI/sapphire substrate.

After LC film casting, photopolymerization was performed for 20 min using a UV lamp. A magnetic field is applied to the sample by $\mathrm{N} 52$ grade $\mathrm{NdFeB}$ magnets during photopolymerization. Figure S2 shows schematic illustration of molecular alignments of LCNs under magnetic fields perpendicular $\left(\mathrm{B}_{\perp}\right)$ and parallel $\left(\mathrm{B}_{\|}\right)$to the substrate. The typical sizes of sample are $1 \times 1 \mathrm{~cm}^{2}$ and $1 \times 0.2 \mathrm{~cm}^{2}$ for perpendicular $\left(\mathrm{B}_{\perp}\right)$ and parallel $\left(\mathrm{B}_{\|}\right)$ field alignments, respectively. The magnetic field strength at the surface of a SmCo is $0.4 \mathrm{~T}$ 
and the magnet field between two SmCo magnets is $0.7 \mathrm{~T}$ as measured by a $\mathrm{DC}$ gaussmeter (AlphaLab, GM-1-ST).

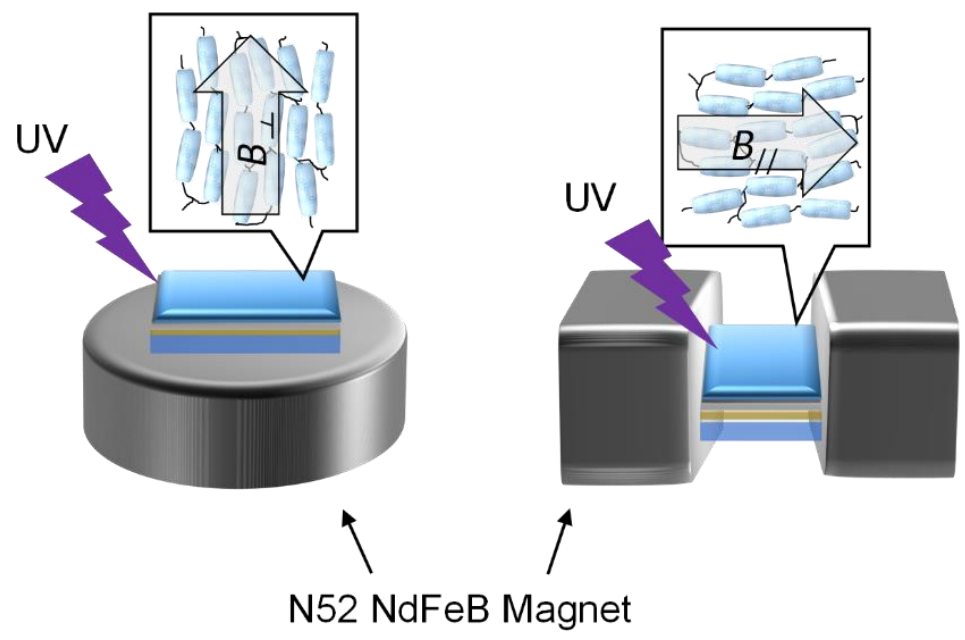

Figure S2. Schematic illustration for molecular alignments of LCNs during UV polymerization of LC solution under perpendicular $\left(\mathrm{B}_{\perp}\right)$ and parallel $\left(\mathrm{B}_{\|}\right)$magnetic fields.

The effect of surface anchoring energy on thermal conductivity was examined by comparing TDTR data between Al films with and without hydrophobic surface functionalization with fluorosilane $(1 \mathrm{H}, 1 \mathrm{H}, 2 \mathrm{H}, 2 \mathrm{H}-$ perfluorodecyltriethoxysilane, Sigma Aldrich). The substrates were soaked in a $10 \mathrm{mM}$ silane toluene solution for $4 \mathrm{~h}$ at room temperature. After that, the substrates were rinsed with toluene and ethanol and dried in a stream of dry N2 gas. The substrates were then heated in an oven at $100{ }^{\circ} \mathrm{C}$ for $1 \mathrm{~h}$.

The sensitivity, $S_{X}$, is calculated from the ratio of the change in a quantity $X$ to a change in the ratio of the in-phase $\left(V_{\text {in }}\right)$ and out-of-phase ( $\left.V_{\text {out }}\right)$ lock-in amplifier signals. $X$ can be any thermal parameter such as thermal conductivity, heat capacity or thickness of each layer. $S_{X}$ is defined as

$$
S_{\mathrm{X}}=\frac{d \ln \left(-\frac{V_{\text {in }}}{V_{\text {out }}}\right)}{d \ln X}
$$

Figure S3A shows a sensitivity plot for our sample geometry. The major factors determining TDTR signal are the thickness and heat capacity of the Al film followed by the thermal conductivity and heat capacity of PI and LCN. 

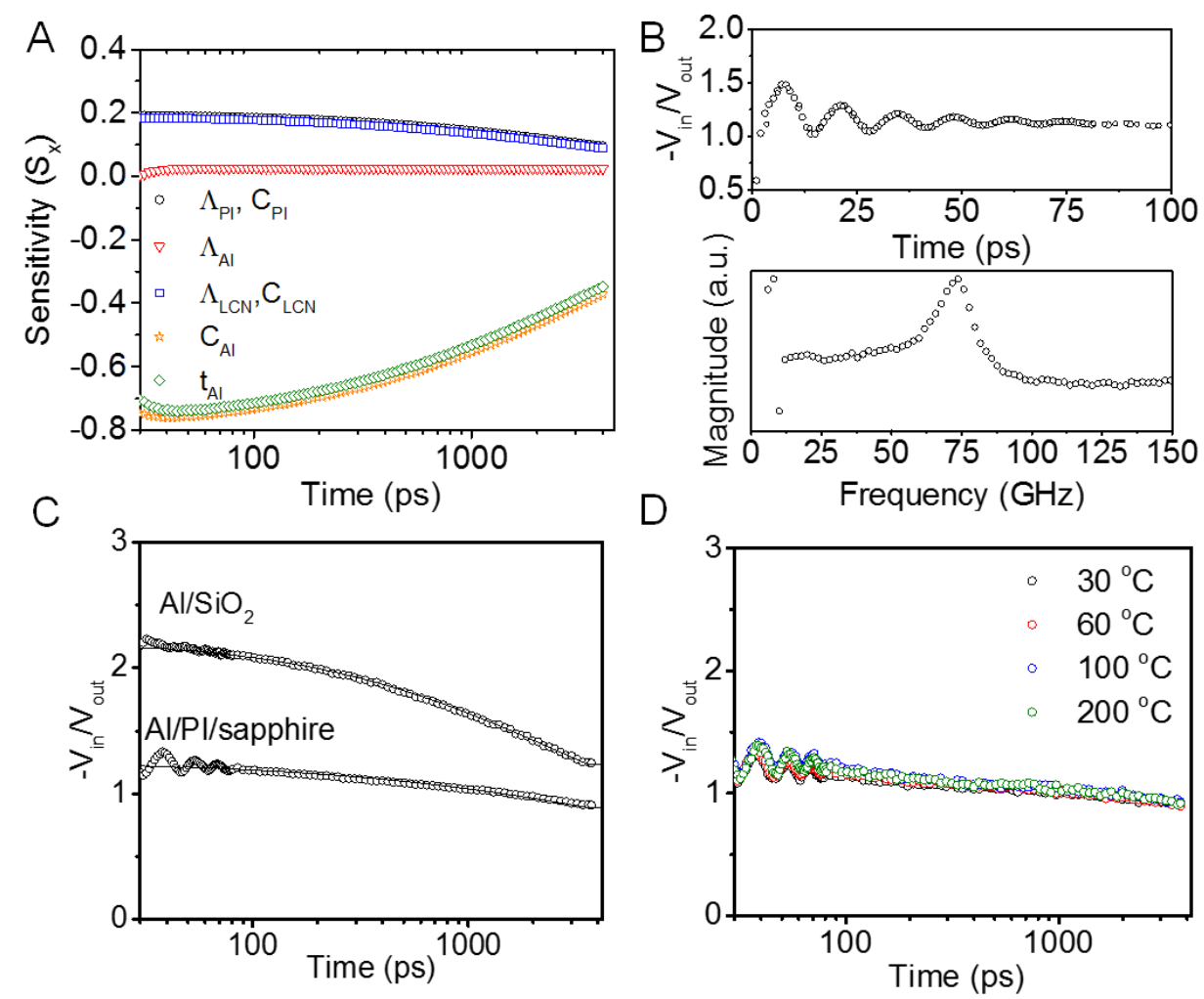

Figure S3. (A) Sensitivity plot for LCN/A1/PI/sapphire sample. (B) Picosecond acoustic signal and Fourier transformed picosecond acoustic signal. (C) TDTR data for $\mathrm{Al} / \mathrm{SiO}_{2}$ and $\mathrm{Al} / \mathrm{PI} / \mathrm{sapphire}$ substrates. (D) TDTR data for A1/PI/sapphire substrate at temperature from $30{ }^{\circ} \mathrm{C}$ to $200{ }^{\circ} \mathrm{C}$.

To fit the data, we measure the thickness of Al by picosecond acoustic signal from TDTR as shown in Figure S3B. The longitudinal acoustic wave generated at the Al/PI interface and and reflected at the other interface $(\mathrm{Al} / \mathrm{LCN})$ by pump beam results in an oscillation frequency of $\Gamma$ :

$$
\Gamma=v_{\mathrm{Al}} / 2 t_{\mathrm{Al}}
$$

where $v_{\mathrm{Al}}$ is the longitudinal speed of sound of $\mathrm{Al}$ and $t_{\mathrm{Al}}$ is the thickness of $\mathrm{Al}$.

The thermal conductivity and heat capacity of $\mathrm{Al}$ were converted from an electric conductivity measured by four point probes and obtained from reference value, respectively. These values were confirmed by fitting TDTR signal from a bare $\mathrm{Al}$ film on a $\mathrm{SiO}_{2}$ substrate as shown in Figure S3C. The thermal property of polyimide (PI) film was obtained from TDTR signal from an Al/PI/sapphire substrate.

To examine thermal stability of the Al/PI/sapphire substrate, we measured TDTR signals of an $\mathrm{Al} / \mathrm{PI} / \mathrm{sapphire}$ substrate up to $200{ }^{\circ} \mathrm{C}$, which is an identical range to the main experiments using LCNs. Figure S3D shows stable signals without notable variations up to 
$200{ }^{\circ} \mathrm{C}$.

The thermal conductivity of RM257 monomers was measured in the presence of magnetic fields perpendicular $\left(\mathrm{B}_{\perp}\right)$ and parallel $\left(\mathrm{B}_{\|}\right)$to the $\mathrm{Al} / \mathrm{PI} / \mathrm{sapphire}$ substrate. First, RM257 monomers were melted at $80{ }^{\circ} \mathrm{C}$ and injected into a cell consisting of an Al/PI/sapphire substrate and glass slide. Then TDTR was performed in the presence of magnetic fields $\left(\mathrm{B}_{\perp}\right.$ and $\mathrm{B}_{\|}$). Figure $\mathrm{S} 4 \mathrm{~A}$ shows the thermal conductivity of RM257 under magnetic fields. Open circles are data points measured by in-situ continuous TDTR with a heating rate of $4{ }^{\circ} \mathrm{C} \mathrm{min}^{-1}$. Open squares are converted from independent TDTR measurements (Figure S4B) carried out at constant temperatures up to $110{ }^{\circ} \mathrm{C}$ in the presence of the perpendicular field $\left(\mathrm{B}_{\perp}\right)$. The thermal conductivity values of RM257 under $B_{\perp}$ and $B$ // were distinguished from each other at above the melting temperature of approximately $65{ }^{\circ} \mathrm{C}$. The anisotropic thermal conductivity of RM257 monomer liquid in the nematic phase were found to be $0.24 \mathrm{~W} \mathrm{~m}^{-1} \mathrm{~K}^{-1}$ and $0.14 \mathrm{~W} \mathrm{~m}^{-1} \mathrm{~K}^{-1}$ at $80{ }^{\circ} \mathrm{C}$ under $\mathrm{B}_{\perp}$ and $\mathrm{B} \|$, respectively.
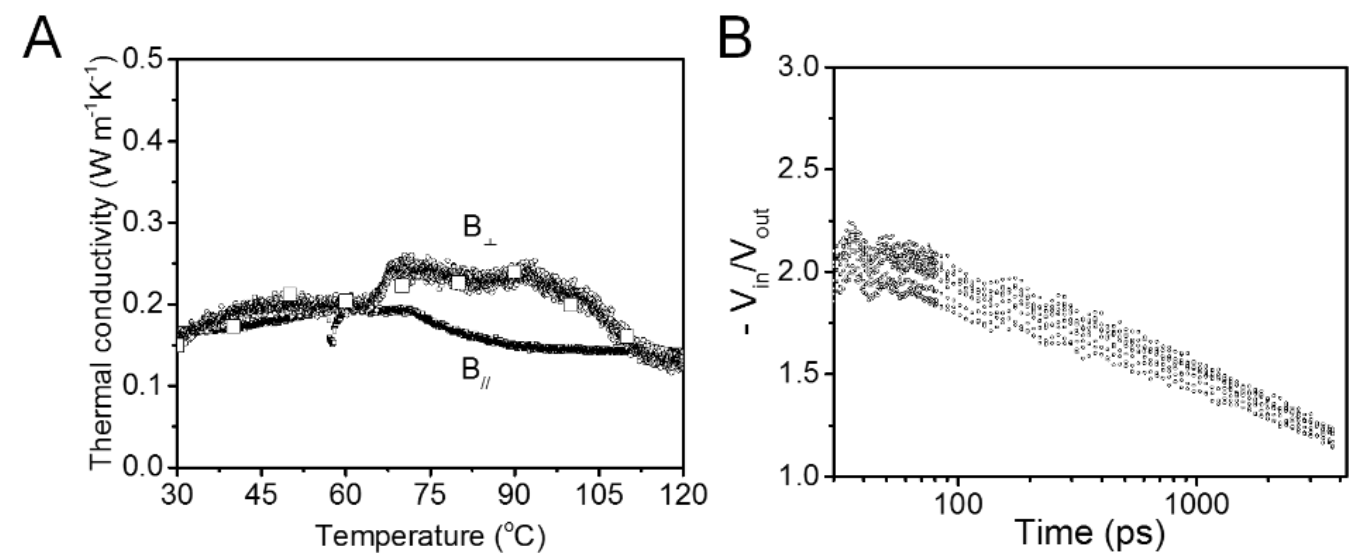

Figure S4. (A) Thermal conductivity of RM257 in the presence of magnetic fields parallel $\left(B_{\|}\right)$and perpendicular $\left(\mathrm{B}_{\perp}\right)$ to the $\mathrm{Al} / \mathrm{PI} / \mathrm{sapphire}$ substrate. (B) Independent TDTR data at fixed temperatures from $30{ }^{\circ} \mathrm{C}$ to $110{ }^{\circ} \mathrm{C}$ in the presence of a perpendicular field $\left(\mathrm{B}_{\perp}\right)$. Thermal conductivity values of each measurement are plotted as open squares in A.

Glancing incident wide angle x-ray scattering (GIWAXS) for LCN with a cross-linker concentration of $20 \mathrm{~mol} \%$ shows a ring pattern which indicates the absence of molecular ordering as shown in Figure S5. The thermal conductivity of LCN with a cross-linker density of $20 \mathrm{~mol} \%$ is $0.2 \mathrm{~W} \mathrm{~m}^{-1} \mathrm{~K}^{-1}$ independent of magnetic field during polymerization. 


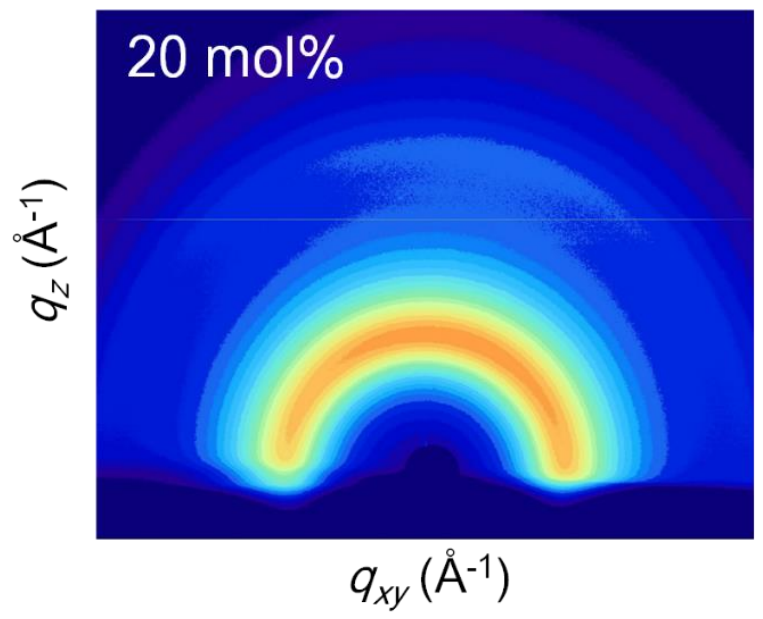

Figure S5. GIWAXS data for LCN with a cross-linker concentration of $20 \mathrm{~mol} \%$. The scattering vector $q\left(2 \pi \lambda^{-1}\right)$ in this figure ranges from $-3 \AA^{-1}$ to $3 \AA^{-1}$ for the $x y$ plane and -0.5 to $4 \AA^{-1}$ for the $z$ plane.

Order parameters for aligned LCNs were obtained from azimuthal intensity $I(\Phi)$ of primary scattering peak of GIWAXS data. ${ }^{1}$

$$
\begin{gathered}
I(\Phi)=\sum_{i=0}^{\infty} f_{2 i} \frac{2^{i} i !}{(2 i+1) ! !} \cos ^{2 i} \Phi \\
f(\beta)=\sum_{i=0}^{\infty} f_{2 i} \cos ^{2 i} \Phi
\end{gathered}
$$

where the orientational distribution function of molecules $f(\beta)$ is used to calculate the order parameter S:

$$
\begin{aligned}
\left\langle\cos ^{2} \varphi\right\rangle & =\frac{\int_{0}^{\pi / 2} f(\beta) \cos ^{2} \beta \sin \beta d \beta}{\int_{0}^{\pi / 2} f(\beta) \sin \beta d \beta} \\
S & =\frac{1}{2}\left(3\left\langle\cos ^{2} \varphi\right\rangle-1\right)
\end{aligned}
$$

where $\varphi$ is the angle between the long axis of the molecules and the magnetic field.

The measured azimuthal intensity $I_{\exp }(\Phi)$ is a sum of $I(\Phi)$ and background intensity $I_{\mathrm{BG}}$. We subtracted $I_{B G}$ from average value from diffuse ring. Figure S6 shows the measured azimuthal intensity $I_{\exp }(\Phi)$ and calculated order parameters from curve fitting (solid line). Small deviations at the edge $\left(\Phi=0^{\circ}\right.$ and $\left.180^{\circ}\right)$ are attributed to shaded area of the detector at lower angles. 
A

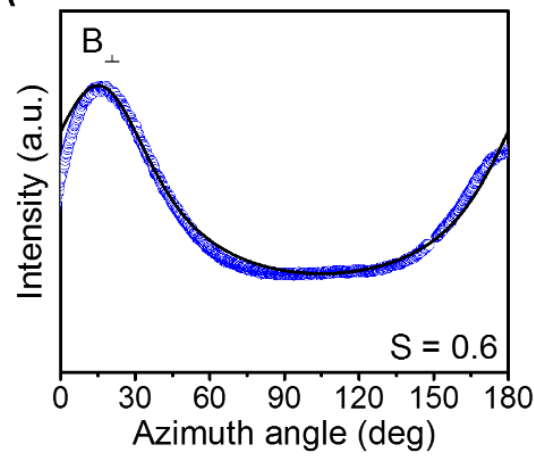

B

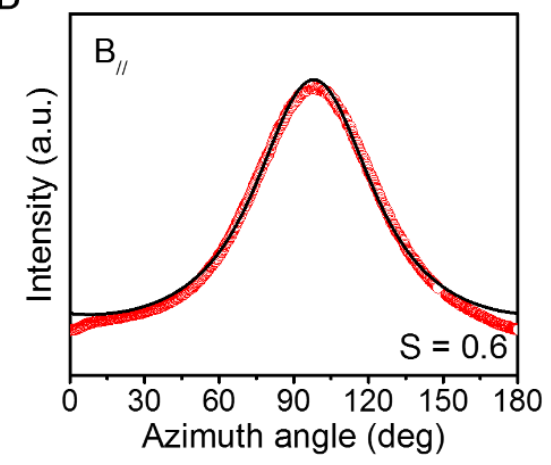

Figure S6. (A) Azimuth intensity of LCNs polymerized in the presence of a magnetic fields perpendicular $\left(\mathrm{B}_{\perp}\right)$ to the substrate. (B) Azimuth intensity of LCNs polymerized in the presence of a magnetic field parallel $\left(\mathrm{B}_{\|}\right)$to the substrate. Order parameters were obtained from curve fitting (solid lines). The corresponding 2-D GIWAXS data is shown in Figure 3 in the main text.

We constructed an effective medium model describing thermal conductivity of LCNs as described in the main text. Figure S7A shows a schematic illustration of an isotropic medium of thermal conductivity $\Lambda_{\text {medium }}$ embedding partially-ordered mesogens with the fundamental in-plane thermal conductivity of $\Lambda_{\text {in-plane }}$ and out-of-plane thermal conductivity of $\Lambda_{\text {out-of-plane. }}$. Figure S7B shows order parameters of magnetically aligned LCNs with different cross-linker concentrations. Figure S7C shows measured thermal conductivity of LCNs with different cross-linker concentrations under magnetic fields and predicted thermal conductivity by the constructed effective medium model.

The thermal conductivity change of $\mathrm{LCN}$ polymerized under $\mathrm{B}_{\perp}$ was measured at various temperatures up to $200{ }^{\circ} \mathrm{C}$. Figure S8 shows a set of TDTR data at fixed temperatures from $25{ }^{\circ} \mathrm{C}$ to $200{ }^{\circ} \mathrm{C}$. The decrease in $-V_{\text {in }} / V_{\text {out }}$ is associated with the thermal conductivity loss as described in the main text. 


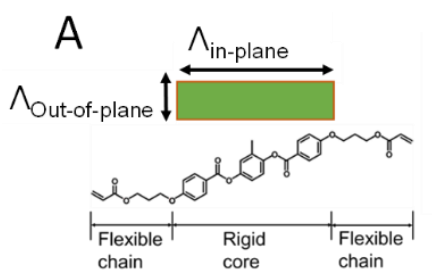

B

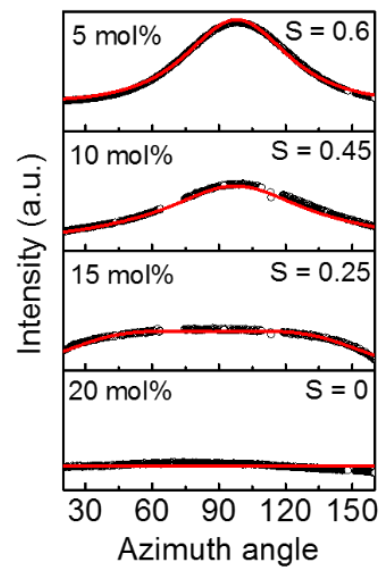

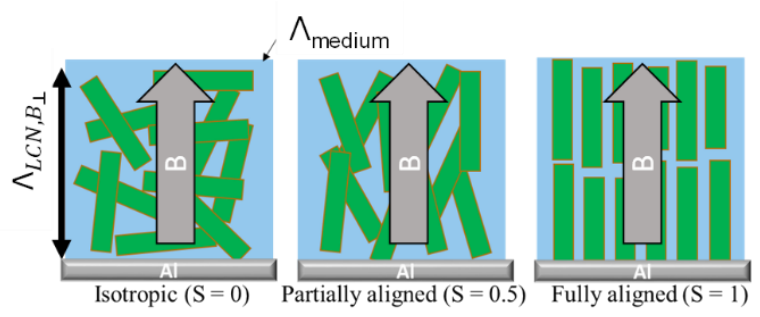

C

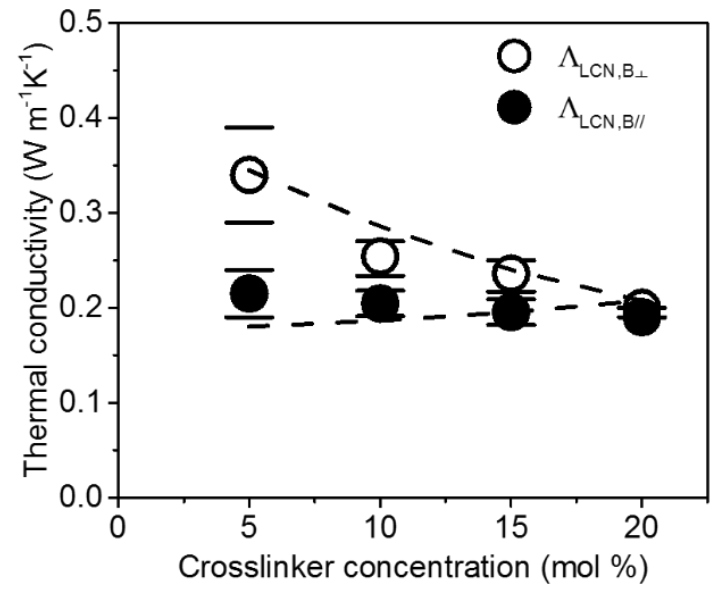

Figure S7. (A) Schematic illustration for effective medium model for partially-ordered, anisotropic cylindrical mesogens with anisotropic thermal conductivity of $\Lambda_{\text {in-plane }}$ and $\Lambda_{\text {out-of-plane }}$ embedded in an isotropic medium with a thermal conductivity of $\Lambda_{\text {medium }}$ (B) The azimuth angle and calculated order parameters for LCNs polymerized with cross-linker concentrations of 5, 10, 15 and $20 \mathrm{~mol} \%$. C. Comparison between thermal conductivity of LCNs polymerized under magnetic fields parallel $\left(\mathrm{B}_{\|}\right)$ and perpendicular $\left(\mathrm{B}_{\perp}\right)$ to the substrate as a function of cross-linker concentration. The dashed line is calculated from the effective medium model.

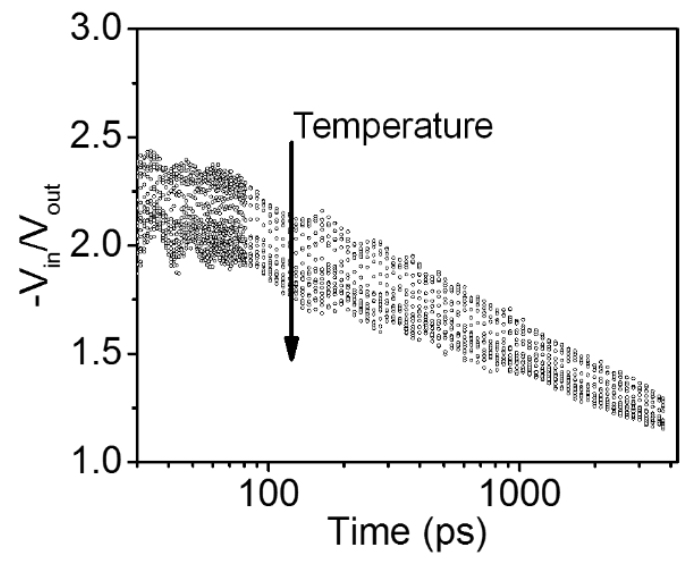

Figure S8. A set of TDTR data for LCN polymerized under $\mathrm{B}_{\perp}$ at fixed temperature from room temperature to $200{ }^{\circ} \mathrm{C}$. Each data points is converted to thermal conductivity values as shown in Figure 4. 
The glass transition temperature of LCN of LCN were characterized by differential scanning calorimetry (Q20 DSC, TA instruments). An endothermic peak marked with an arrow in Figure S9 indicates the glass transition temperature at $65{ }^{\circ} \mathrm{C}$, which is in the typical range of $40-120{ }^{\circ} \mathrm{C}$ for $\mathrm{LCNs}^{2}$

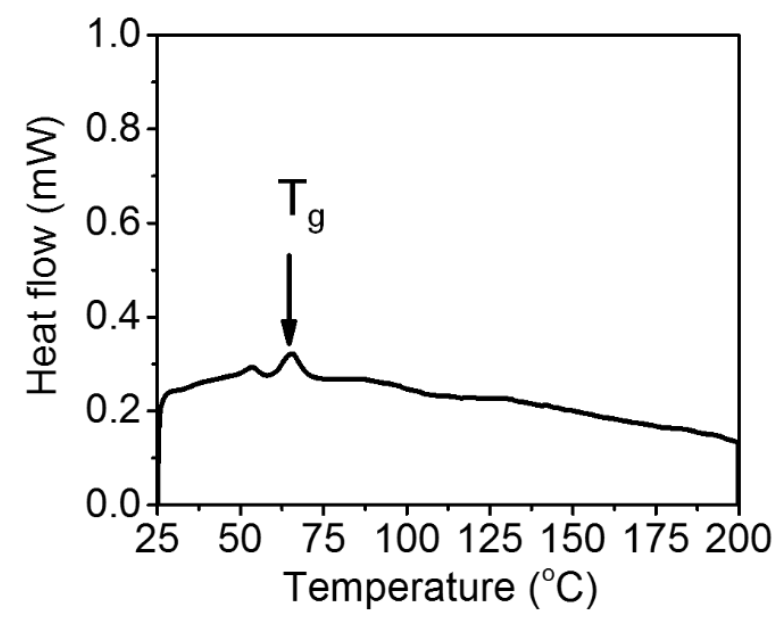

Figure S9. DSC analysis of LCN with a scanning rate of $4{ }^{\circ} \mathrm{C} \min ^{-1}$.

As a further test for residual nematic ordering in LCNs, we carried out transmittance WAXS on bulk LCNs samples. We prepared bulk LCNs samples by filling a LC solution with a 0,5 and $20 \mathrm{~mol} \%$ cross-linker in a capillary tube followed by UV polymerization at nematic $\left(80{ }^{\circ} \mathrm{C}\right)$ and isotropic $\left(140{ }^{\circ} \mathrm{C}\right)$ temperatures.

Figure S10 shows that bulk LCNs at nematic temperature $\left(80^{\circ} \mathrm{C}\right)$ and isotropic temperature $\left(140{ }^{\circ} \mathrm{C}\right)$ exhibit isotropic ring patterns in WAXS measurements without any specific ordering peaks. A slight decrease in diffraction intensity was observed for LCNs prepared at $140{ }^{\circ} \mathrm{C}$ compared to $80{ }^{\circ} \mathrm{C}$.

The bulk isotropic x-ray patterns are identical to those obtained for thin film LCNs after thermal disordering as shown in Figure 5b or thin film LCNs prepared with $20 \mathrm{~mol} \%$ crosslinker as shown in Figure S5. Although the LCNs in this study comprise a substantial amount of RM257 monomer, we observed that they evolve to an isotropic state without displaying any residual ordering. 


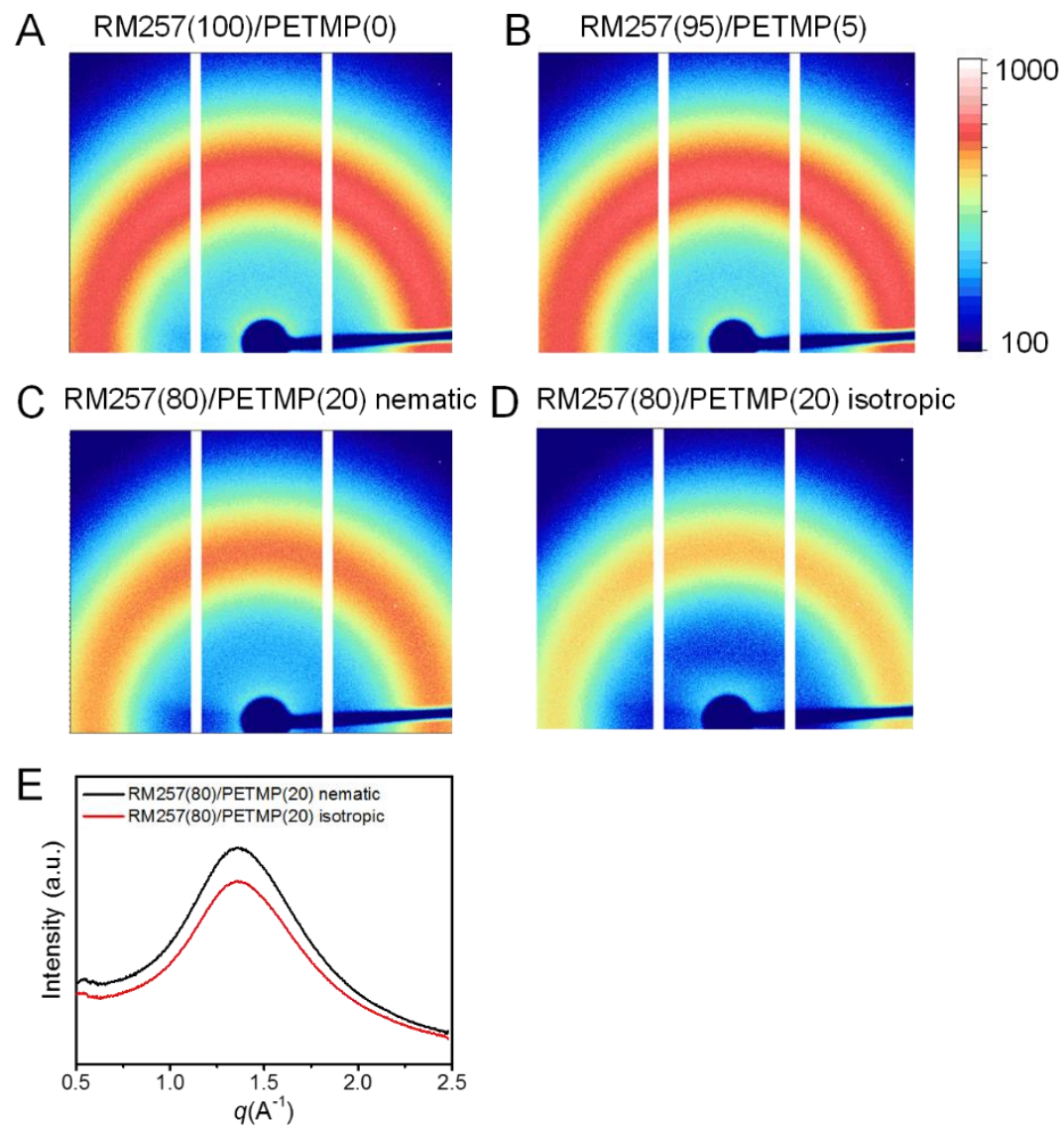

Figure S10. WAXS data for bulk LCNs prepared with a molecular ratio of RM257 to PETMP of (A) 100:0, (B) 95:5, and (C) 80:20 at nematic temperature $\left(80{ }^{\circ} \mathrm{C}\right.$ ). (D) WAXS data for bulk LCNs prepared with a molecular ratio of RM257 to PETMP (80/20) at isotropic temperature $\left(140{ }^{\circ} \mathrm{C}\right)$. (E) Integrated WAXS intensity of bulk LCNs prepared with a molecular ratio of RM257 to PETMP of $80 / 20$ at $80{ }^{\circ} \mathrm{C}$ and $140{ }^{\circ} \mathrm{C}$.

The thermal conductivity change of LCN can be suppressed in the presence of magnetic field. Figure S11 shows that the thermal conductivity of LCNs polymerized under $\mathrm{B}_{\|}$and $\mathrm{B}_{\perp}$ at temperatures up to $200{ }^{\circ} \mathrm{C}$. Contrary to the thermal conductivity decrease of LCN polymerized under $\mathrm{B}_{\perp}$ without magnetic field, $\mathrm{LCN}$ polymerized under $\mathrm{B}_{\perp}$ exhibits a slight increase in the thermal conductivity up to $150{ }^{\circ} \mathrm{C}$ (Blue dots). Likewise, LCN polymerized under $\mathrm{B} \|$ exhibits a slight decrease in the thermal conductivity up to $150{ }^{\circ} \mathrm{C}$. 


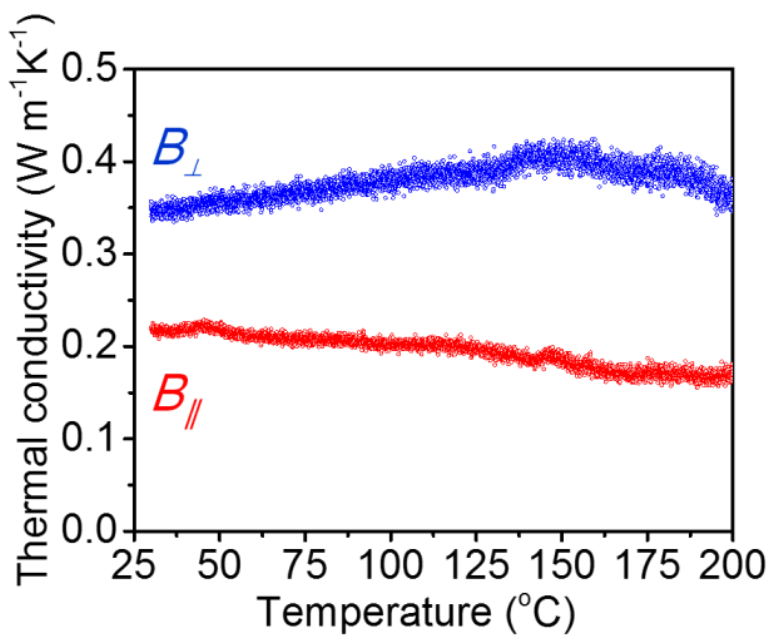

Figure S11. Thermal conductivity of LCN polymerized in the presence of magnetic fields perpendicular $\left(\mathrm{B}_{\perp}\right)$ and parallel $\left(\mathrm{B}_{\|}\right)$to the $\mathrm{Al} / \mathrm{PI} /$ sapphire substrate.

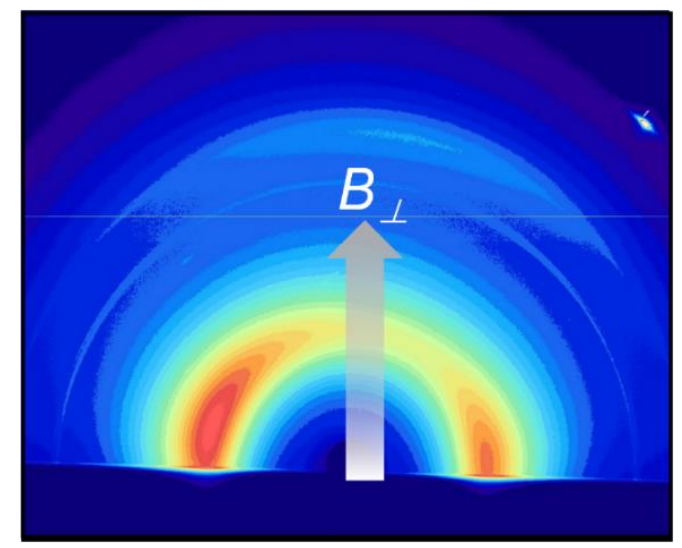

Figure S12. GIWAXS pattern after magnetic rotation. The magnetic field was changed from parallel $\left(\mathrm{B}_{\|}\right)$to perpendicular $\left(\mathrm{B}_{\perp}\right)$ direction at elevated temperatures of $200{ }^{\circ} \mathrm{C}$ and slowly cooled to the room temperature. This image was taken at room temperature after the magnetic rotation. The scattering vector $q\left(2 \pi \lambda^{-1}\right)$ in this figure ranges from $-3 \AA^{-1}$ to $3 \AA^{-1}$ for the $x y$ plane and -0.5 to $4 \AA^{-1}$ for the $z$ plane.

The rheological characterization of LCNs was carried out by a rotational rheometer (ARG2, TA instruments) with coin-shaped LCN samples ( $25 \mathrm{~mm}$ in diameter, $1 \mathrm{~mm}$ in thickness). The measurement was done in a linear viscoelastic regime with an oscillation strain of $1 \%$ and frequency of $10 \mathrm{rad} \mathrm{s}^{-1}$. Samples show a slight variation about $\sim 35 \%$ in the storage modulus G'. From equation $M_{\mathrm{c}}=\rho R T \mathrm{G}^{-1}$ changes in storage modulus also indicate variations in the average distance between cross-linkers of $\sim 14$ units of RM257, where $M_{\mathrm{c}}$ is the average molecular weight between cross-linkers, $\rho$ is the density, $R$ is the gas constant and $T$ is the temperature. The dynamic viscosity measured by the oscillatory rheometer is also used to calculate the relaxation time as discussed in the main text. 

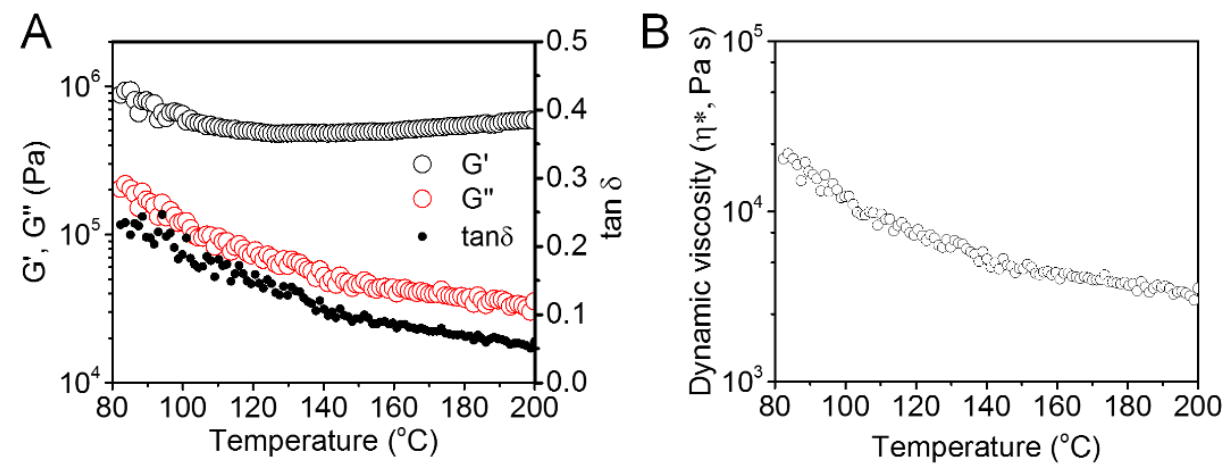

Figure S13. Storage modulus G', loss modulus G", and $\tan \delta$ of a LCN sample. Dynamic viscosity of a LCN measured by the oscillatory rotational rheometer.

1. Davidson, P.; Petermann, D.; Levelut, A. M. J. Phys. Ii. 1995, 5 (1), 113-131.

2. White, T. J.; Broer, D. J. Nat. Mater. 2015, 14 (11), 1087-1098. 